4 10 P.Jarvis ${ }^{1}$; I.Guymer ${ }^{3}$; Bedford, MK43 OAL, UK

${ }^{2}$ Department of Geography, University of Leicester, Leicester, LE1 7RH, UK

${ }^{3}$ School of Engineering, University of Warwick, UK

\title{
A multi-component method to determine pesticides in surface water by liquid-chromatography tandem quadrupole mass spectrometry
}

A.M.Ramos ${ }^{1} ;$ M.J.Whelan ${ }^{2} ;$ S.Cosgrove $^{1} ;$ R.Villa $^{1} ;$ B.Jefferson $^{l} ;$ P.Campo $^{1 *}$;

${ }^{1}$ Water Science Institute, School of Energy, Environment and Agrifood, Cranfield University, 


\section{Abstract}

26 Pesticide pollution of surface water is a major concern in many agricultural catchments. In the 27 EU, water companies are required to supply water to their customers with concentrations of 28 individual pesticides no higher than $0.1 \mu \mathrm{g} \mathrm{L} \mathrm{L}^{-1}$. However, concentrations in untreated water frequently exceed this limit for a number of different pesticides leading to occasional compliance challenges. The development of rapid and accurate methods for determining pesticide concentrations in water samples is, therefore, important. Here we describe a method for the simultaneous analysis of six pesticides (metaldehyde, quinmerac, carbetamide, metazachlor, propyzamide and pendimethalin) in natural waters by direct aqueous injection

34 with liquid chromatography-tandem mass spectrometry. The method validation showed good linearity from 0.2 to $50.0 \mu \mathrm{g} \mathrm{L}^{-1}$ with correlation coefficients between 0.995 to 0.999 . Method accuracy ranged from 84 to $100 \%$ and precision (RSD) from 4 to $15 \%$. The limits of detection for the targeted pesticides ranged from 0.03 to $0.36 \mu \mathrm{g} \mathrm{L}^{-1}$. No significant matrix effects on quantification were observed ( $t$ test). The method was tested on water samples from a small arable catchment in eastern England. Peak concentrations for the determinands ranged

40 from 1 to $10 \mu \mathrm{g} \mathrm{L}^{-1}$.

\section{Key Words}




\section{1. Introduction}

54 The use of pesticides is a necessary part of modern agriculture in order to keep up with increasing demands for food, fibre and energy from the growing global population. Nevertheless, pesticides can be transported from land to ground and surface waters by spray drift, leaching and surface runoff, where they can pose problems for aquatic ecosystems and for the quality of abstracted drinking water [1].

Agriculture is generally considered to be the greatest contributor to pesticide pollution in many ground and surface waters, although in some catchments runoff from hard surfaces may be locally important [2]. In the European Union (EU), the Drinking Water Directive (DWD: 98/83/EC) sets a limit for the concentration of individual pesticide active ingredients in drinking water at $0.1 \mu \mathrm{g} \mathrm{L}^{-1}$ and a limit of $0.5 \mu \mathrm{g} \mathrm{L}^{-1}$ for the detection of multiple pesticides[1]. To better understand the compliance risks, water companies and environmental regulatory agencies need to monitor abstracted water bodies, as well as treated water being supplied to consumers. Pesticide monitoring is a challenging task because a high number of active ingredients is typically used in catchments with mixed land use (presenting a wide range of physico-chemical properties) which are applied at different times of year and at different rates. This means that several different analytical methods may need to be employed on a single sample in order to detect the compounds of interest. The challenges of detecting target compounds can also be exacerbated by the episodic nature of pesticide transport from land to water (which tend to occur predominantly during storm events) [3]. Hence, high sampling frequencies may be required to capture representative temporal patterns, which results in significant analytical costs.

Most methods for pesticide analysis at the low concentrations generally encountered in natural water bodies require a sample pre-concentration step such as solid phase extraction (SPE), solid phase micro-extraction, or liquid-liquid extraction. Of these techniques, SPE is most commonly employed because it often provides good sample extraction, concentration and clean up[4][5]. However, there are several disadvantages with this technique including potential for low recoveries, long processing times per sample, the high cost of SPE cartridges and differing extraction procedures for different classes of pesticide owing to their 82 polarities.

83 As an alternative, direct aqueous injection (DAI) methods have been developed for the analysis of a wide range of pesticides in various sample matrices. Applications include analysis of polar organophosphorus pesticides in fruit and vegetables [6] and analysis of 
pesticides in potable water [7]. The main advantages of DAI are easy sample preparation/ manipulation, low consumable costs and reduced analysis time allowing high sample throughput as well as low limits of detection $\left(<0.1 \mu \mathrm{g} \mathrm{L}^{-1}\right)$.

In this paper, we describe a DAI multi-component method for the determination of six pesticides by LC-MS/MS in environmental waters. The specific requirements of the method were to be accurate and rapid so as to allow the efficient processing of a large number of samples. The pesticides analysed were metaldehyde, quinmerac, metazachlor, carbetamide, propyzamide and pendimethalin. Molecular structures and relevant physico-chemical properties are listed in Table 1. With the exception of pendimethalin, all the compounds examined have organic carbon-water partition coefficient $\left(K_{o c}\right)$ values less than $217 \mathrm{~L} \mathrm{~kg}^{-1}$, which suggests that they will be moderately mobile in soil and, hence, prone to leaching losses. All six pesticides are widely used in arable agriculture in Europe and have been previously detected at concentrations of concern in UK water bodies [3][8]. Metaldehdye is a particular problem for the UK water industry and has been responsible for the highest number of compliance failures in recent years [9][10]. It is a selective molluscicide which is widely used to control slugs and snails in several crops. It is only moderately mobile $\left(K_{o c}=240 \mathrm{~L} \mathrm{~kg}^{-}\right.$ ${ }^{1}$ ) and has been observed to degrade in water-sediment interface with a median dissipation time $\left(D T_{50}\right)$ of 12.2 days (Table 1) which should, in principle reduce the risk of leaching loss from soil. Nevertheless, it has a very stable structure which means that it is difficult to remove in drinking water treatment (typically employing sand filtration, granulated activated carbon filtration, ozonation and or chlorination) [9].

Quinmerac is used to control Galium aparine, Veronica spp and other broad leaved weeds in cereals, oil seed rape and sugar beet. Carbetamide and propyzamide are herbicides used to control black grass infestations predominantly in oil seed rape [11]. Metazacahlor and pendimethalin are also herbicides used to control grass and broad-leaved weeds in a range of crops including oil seed rape and Brussel sprouts [11]. Pendimethalin is not expected to be particulary mobile and was included to provide a contrast to the other more mobile compounds.

114 There are few published papers that report on the analysis of more than one of our target 115 pesticides. In general, these protocols only included 2 or 3 pesticides at the most with fruits 116 and vegetables being the studied matrices. Analysis in food stuffs requires an extraction step 117 before any determination can take place. A popular method is QuEChERS which includes 118 SPE followed by LC-MS/MS. Pesticides detected by this method include metazachlor, 119 pendimethalin and quinmerac [12], [13]. Others used homogenisation followed by 
120 evaporation or supercritical fluid extraction as the extraction step followed by GC-MS or GC-

121 NPD (Nitrogen, Phosphorus Detection). Pesticides detected following these methods included 122 carbetamide, propyzamide and pendimethalin [14], [15]. Other protocols dealt with several of 123 our target pesticides in water samples, namely carbetamide, metazachlor, propyzamide [16] 124 metazachlor and pendimethalin [17]. These protocols involved SPE followed by LC-MS and 125 GC-MS retraspectively, although the method by Irace-Guigand (2004) required additional 126 UV-DAD detection.

128 Of the six target pestices, metaldehyde appears to be one of the more difficult compounds to 129 detect in complex samples containing several analytes. In fact, only one paper has reported 130 the analysis of metaldehyde in a multiresidue method alongside any of our target pesticides 131 (i.e. propyzamide) [18]. This approach used SPE prior to GC-MS for food extracts. To the 132 best of our knowledge, no method has been previously reported for the combined rapid 133 determination of these particular six pesticides with minimal sample preparation approach in 134 environmental water samples. 
137 Table 1. Physico-chemical properties for the pesticides considered in this method.

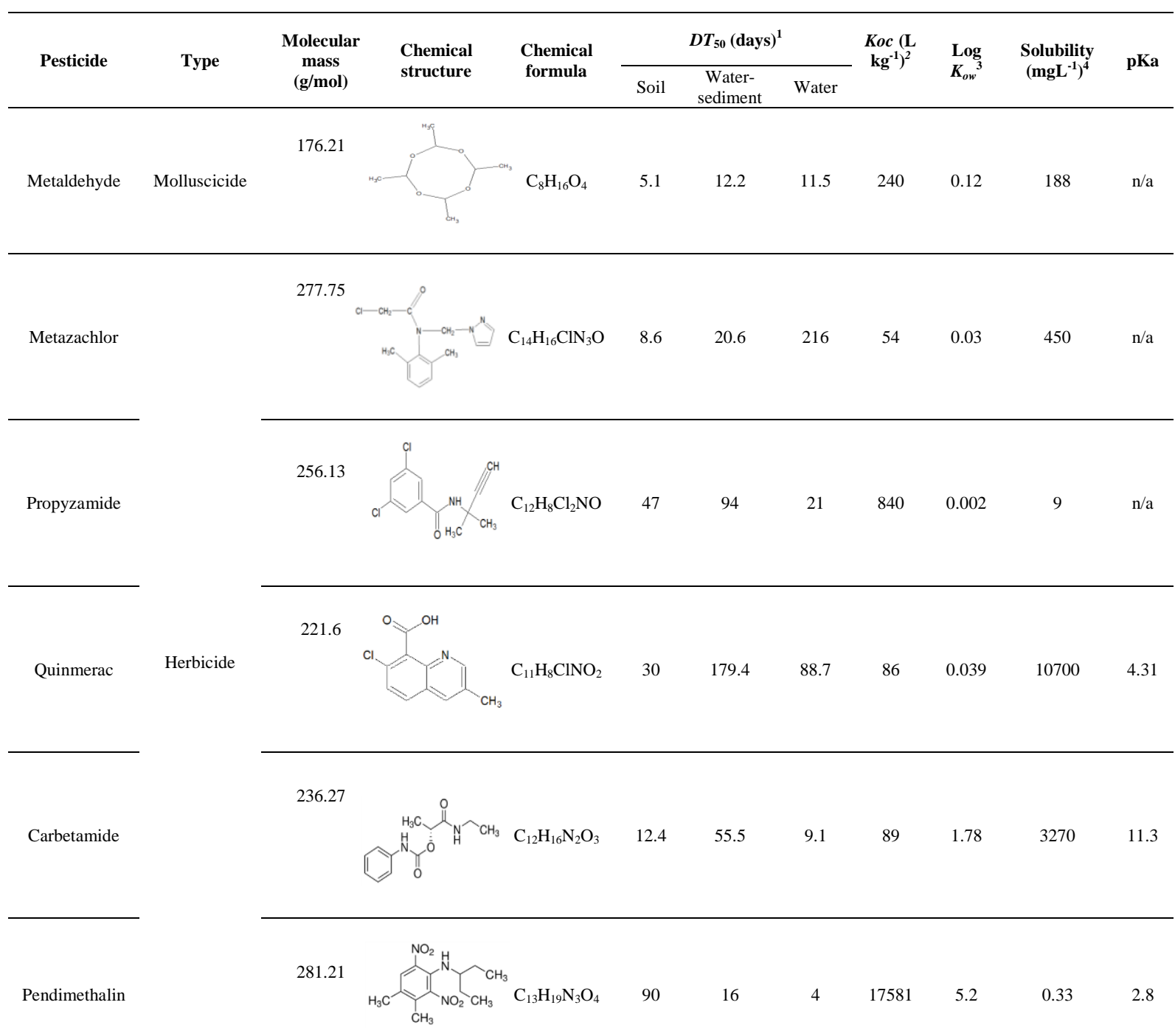

$138{ }^{\mathrm{T}} D T_{50}-$ Median dissipation time in different test systems; ${ }^{2} K_{o c}-$ organic carbon-water partition coefficient $(\mathrm{L} \mathrm{kg}-$

$\left.139{ }^{1}\right) ;{ }^{3} \log K_{o w}-$ octanol-water partition coefficient; ${ }^{4}$ Solubility in water $\left(\mathrm{mg} \mathrm{L}^{-1}\right)$ [19] 


\section{2. Experimental}

\section{2.1. Chemicals and reagents}

143 Pesticide standards were purchased from QMX laboratories (UK), methanol (HPLC grade) 144 and acetic acid (HPLC grade) were obtained from Sigma-Aldrich (UK). Ultra pure water was 145 produced by PURELAB ${ }^{\circledR}$ ultra, Elga.

\section{$146 \quad$ 2.2. Standards and stock solutions}

147 Pesticide stock solutions $\left(100 \mu \mathrm{g} \mathrm{L}^{-1}\right)$ were prepared by dissolving the neat pesticides in 148 methanol. Working standards were prepared by diluting with ultra pure water with 149 concentrations of $0.2,0.5,1.0,2.0,5.0,8.0$ and $10.0 \mu \mathrm{g} \mathrm{L}^{-1}$ for each pesticide. All standards 150 were stored at $4{ }^{\circ} \mathrm{C}$ for a maximum of one month.

\section{$151 \quad$ 2.3. Instrumentation}

152 All analyses were performed with a Waters Alliance 2695 liquid-chromatography system 153 coupled to a Quattro premier XE tandem quadrupole. A Kinetex C18 column $(5 \mu \mathrm{m} 150 \times 2.1$ $154 \mathrm{~mm}$, Phenomenex, UK) thermostated at $60{ }^{\circ} \mathrm{C}$ was used for chromatographic separation. The 155 flow rate was $0.3 \mathrm{~mL} \mathrm{~min}^{-1}$ and the injection volume was $50 \mu \mathrm{L}$. The mobile phase consisted 156 of ultra-pure water with $0.1 \%$ acetic acid (A) and methanol with $0.1 \%$ acetic acid (B). The 157 elution started at $10 \%$ B and was linearly increased to $98 \%$ over 12 min, then maintained for

1583 min before returning to the intital composition. The total time of analysis per sample was 18 159 min.

160 Operating conditions of the mass spectrometer were optimized by infusion of each individual 161 pesticide at a concentration of $1 \mathrm{mg} \mathrm{L}^{-1}$ in a solution of $70 \% \mathrm{~A}$ and $30 \% \mathrm{~B}$. Electrospray 162 ionization (ESI) was performed in positive mode. The mass spectrometer was operated under 163 multiple reaction monitoring (MRM) with two reactions monitored for each analyte (Table 2), 164 with the exception of metaldehyde, which forms a $\mathrm{Na}^{+}$adduct and its fragmentation $165[\mathrm{M}+\mathrm{Na}]^{+}$showed a reaction whose precursor and fragment ions were $\mathrm{m} / \mathrm{z}, 198.9$ and $\mathrm{m} / \mathrm{z} 66.9$, 166 respectively. The UK Environment Agency recommends this reaction for quantitative 167 purposes [18]. 
169 Table 2. SRM transitions used for target compounds.

\begin{tabular}{c|c|c|c|c|c|c|c|c|c}
\hline \multirow{2}{*}{ Analytes } & \multicolumn{3}{|c|}{$\mathbf{1}^{\text {st }}$ transition - quantification } & \multicolumn{2}{c|}{$\mathbf{2}^{\text {nd }}$ transition - confirmation } & Retention \\
\cline { 2 - 11 } & $\begin{array}{c}\text { Percursor } \\
\text { ion } \\
(\boldsymbol{m} / \boldsymbol{z})\end{array}$ & $\begin{array}{c}\text { Product } \\
\text { ion } \\
(\boldsymbol{m} / \boldsymbol{z})\end{array}$ & $\mathbf{c o n e}$ & collision & $\begin{array}{c}\text { Percursor } \\
\text { ion } \\
(\boldsymbol{m} / \boldsymbol{z})\end{array}$ & $\begin{array}{c}\text { Product } \\
\text { ion } \\
(\boldsymbol{m} / \boldsymbol{z})\end{array}$ & cone & $\begin{array}{c}\text { collision } \\
(\mathbf{m i n})\end{array}$ \\
\hline Metaldehyde & 198.9 & 66.9 & 25 & 12 & - & - & - & - & 5.69 \\
\hline Quinmerac & 222.3 & 204.3 & 30 & 25 & 222.3 & 176.3 & 30 & 25 & 6.57 \\
\hline Carbetamide & 237.1 & 191.9 & 15 & 10 & 237.1 & 117.9 & 15 & 8 & 7.85 \\
\hline Metazachlor & 278.1 & 133.8 & 15 & 15 & 278.1 & 209.9 & 15 & 15 & 9.43 \\
\hline Propyzamide & 256.0 & 189.9 & 15 & 15 & 256.0 & 172.8 & 15 & 15 & 10.37 \\
\hline Pendimethalin & 282.1 & 212.0 & 25 & 10 & 282.1 & 193.9 & 25 & 25 & 12.59 \\
\hline
\end{tabular}

170

\section{$171 \quad$ 2.4. Sample collection and Analysis}

172 The method was tested on samples collected from a monitoring study in a small headwater

173 stream at Hope farm in Knapwell, Cambridgeshire, UK (Figure 1). The stream drains a low

174 relief catchment (elevation range 41-78 m above mean sea level) of approximately $3.9 \mathrm{~km}^{2}$,

175 which is dominated by arable land. 


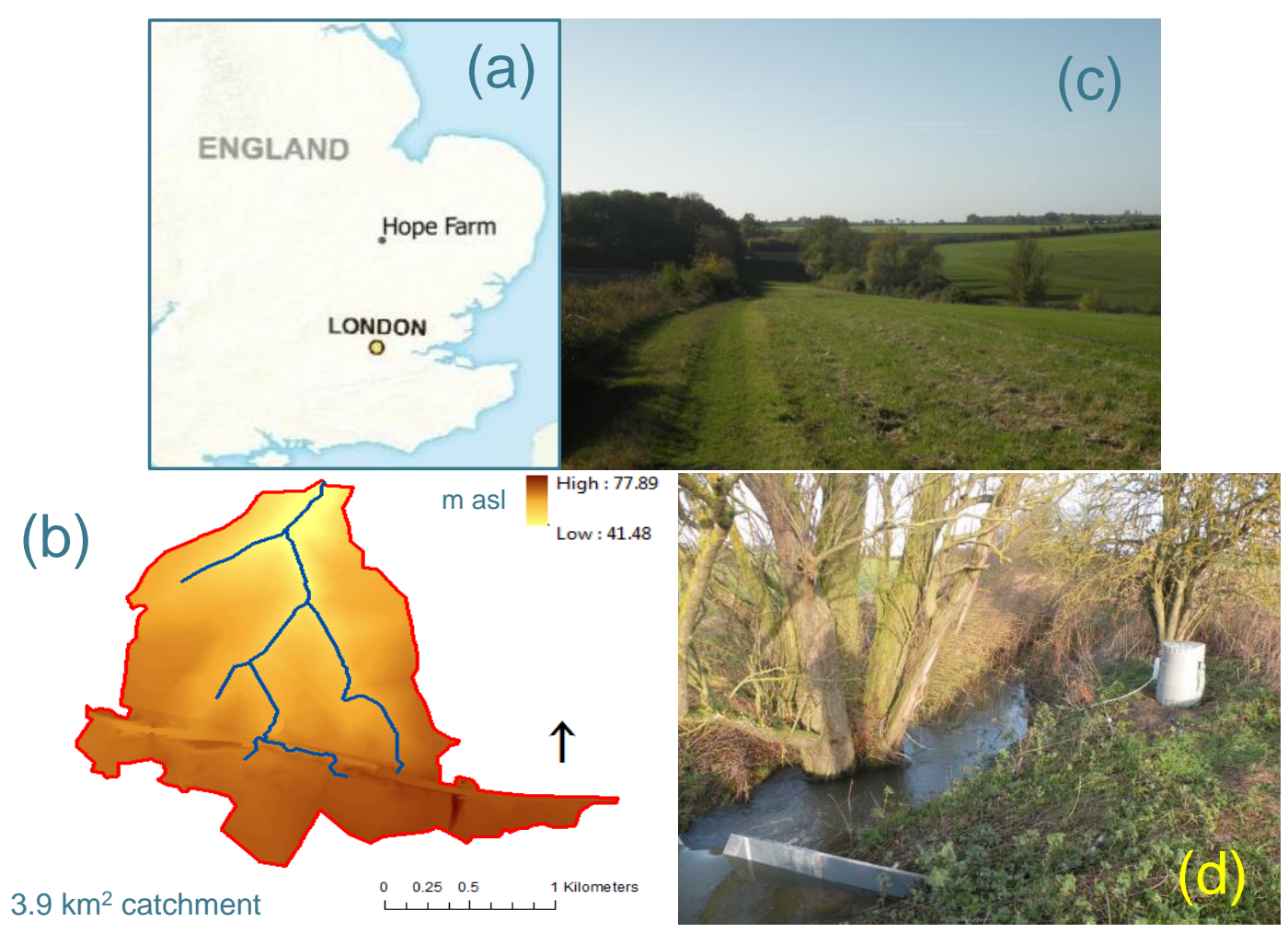

180 Figure 1. (a) Location of study catchment ; (b) Catchment boundary, stream network and digital elevation model; (c) Catchement relief looking upstream ; (d) Automatic water sampler and v-notch weir installed at the catchment outlet.

184 The predominant crop rotation is wheat-oil seed rape and most of the soils belong to the 185 Hanslope Soil Association, which is a typically under-drained. Stream discharge is low (but usually perennial) in summer, which suggests minimal baseflow contributions and is flashy in winter with flows often exceeding $150 \mathrm{~L} \mathrm{~s}^{-1}$ during storm events. The stream was monitored for five months between August 2014 and December 2015. Discharge was measured with a $90^{\circ}$ v-notch weir, equipped with an ISCO AV2150 water level and a velocity sensor. Samples were collected with an ISCO 6712 automatic water sampler at constant sampling intervals of

$1918 \mathrm{~h}$, with a sample volume of $250 \mathrm{~mL}$. Sample bottles were changed approximately every 7 days and replaced with fresh bottles which had been thoroughly pre-cleaned before each change-over using water and methanol. Pesticide concentrations in field bottle blanks,

194 prepared with ultra pure water, were always less than the limits of detection (LOD) and often not detectable. Samples were refrigerated immediately upon arrival to the laboratory 
196 (typically less than $2 \mathrm{~h}$ after sample collection) and filtered through $0.2 \mu \mathrm{m}$ syringe-mounted 197 disc filters (Milipore Millex ${ }^{\mathrm{TM}}$, Fisher Scientific, UK) within 24 h of collection.

\section{2.5. Sample injection and data processing}

200 Sample runs consisted of eight working standards, followed by five unknown samples with 201 solvent blanks and continuing calibration checks $\left(5 \mu \mathrm{g} \mathrm{L}^{-1}\right)$ in between. Runs never exceeded 20280 determinations including analytical standards, blanks, calibration checks and samples. 203 Peak areas of target pesticides were obtained with Quantlynx v.4.1. Weighted (1/x) linear 204 least-squares regression curves were fitted to the observations and not forced through the 205 origin. 


\section{Results and discussion}

208 Figure 2 shows an example total ion chromatogram (TIC) for the six pesticides in positive ion mode analysed over $18 \mathrm{~min}$ from a $10 \mu \mathrm{g} \mathrm{L}^{-1}$ standard of each pesticide in ultra-pure water.

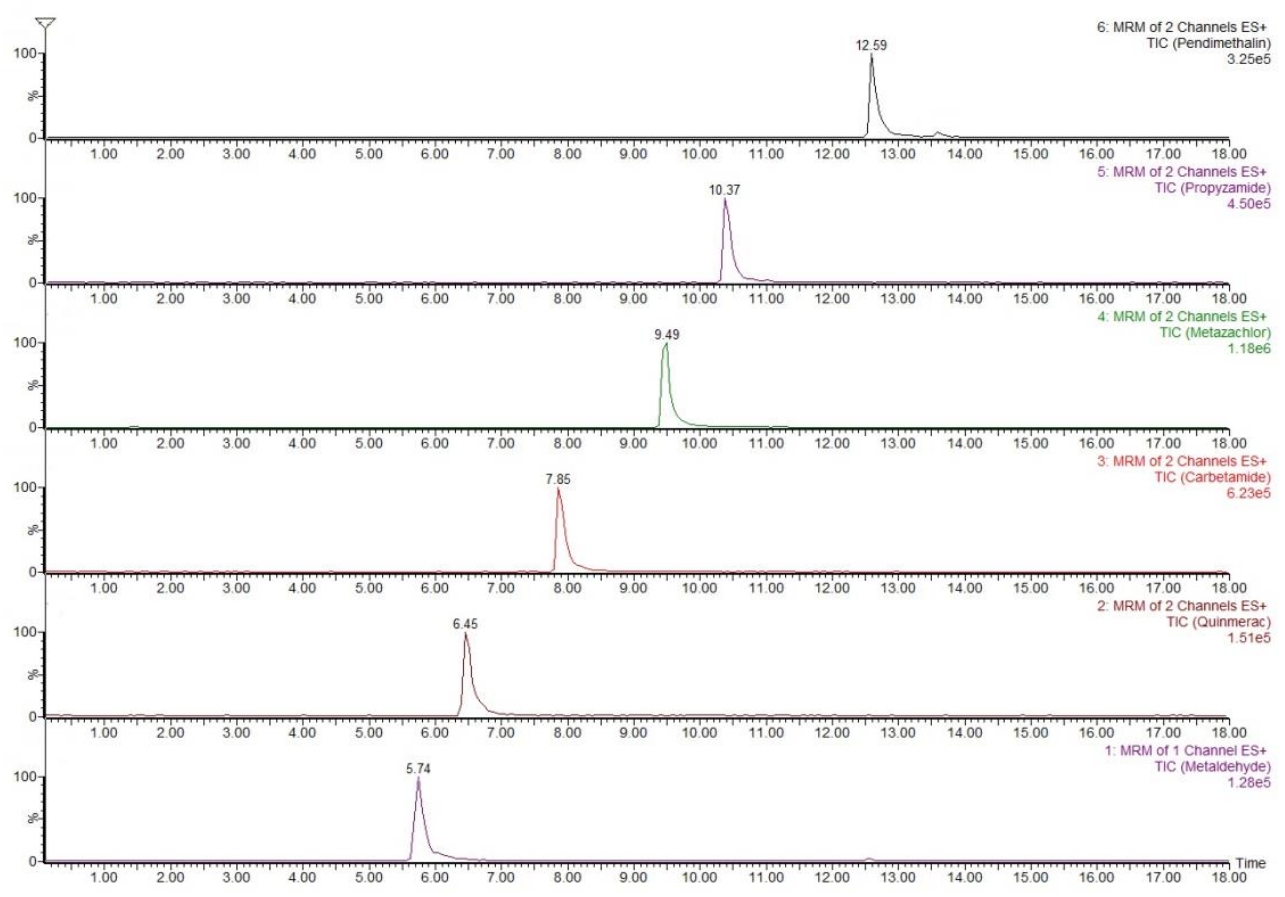

213 Figure 2. Example chromatograms of six pesticides at $10 \mu \mathrm{g} \mathrm{L}^{-1}$ in ultra-pure water by direct aqueous injection.

\subsection{Optimisation of the MS/MS parameters.}

216 For the MS operation, only ESI in positive mode was evaluated for the determination of the

217 six pesticides. The optimum cone voltage and collision energies are reported in Table 2. Good 218 peak shape and suitable signal-to-noise ratios were obtained with a dwell time of $0.25 \mathrm{~s}$. 


\subsection{Optimisation of the LC conditions}

222 Optimisation of mobile phase composition and elution gradient was very important to achieve 223 good separation, high sensitivity, good ionization and resolution, particularly for trace analysis. Results (see example in Figure 2) showed that higher sensitivity and good peak shape could be achieved with $0.1 \%$ acetic acid in both eluents. The gradient was optimised to obtain improved resolution and shorter analysis time.

\subsection{Validation procedures}

The analytical method was validated according to the performance criteria established by ICH guidelines [19]. The validation parameters evaluated were linearity, accuracy, precision, LODs, limits of quantification (LOQs) and matrix effect.

\subsubsection{Linearity}

234 Method linearity was evaluated by analysing the response for the seven concentration levels 235 prepared from the working standard solution described in Section $2.2(0.2,0.5,1.0,2.0,5.0$, 2368.0 and $10 \mu \mathrm{g} \mathrm{L}^{-1}$ ). Linear regression analysis of calibration data was performed by plotting the peak areas of the quantitative ion versus the corresponding standard concentrations. Good linearity was achieved with coefficients of determination between 0.994 to 0.999 (Table 3). The method provided acceptable precision, accuracy and linearity over the range of 0.2 to $50.0 \mu \mathrm{g} \mathrm{L}^{-1}$.

\subsubsection{Accuracy and Precision}

243 Inter-day and intra-day accuracy and precision (RSD) were assessed. Inter-day comparisons

244 express within laboratory across-day variations while intra-day comparisons express within 245 laboratory within-day variations. The intra-day test consisted of five consecutive analyses, 246 while the inter-day variations were assessed on different days for a $5 \mu \mathrm{g} \mathrm{L}^{-1}$ standard. Intra247 day precision (RSD) varied from $17.4 \%$ (pendimethalin) to $3.1 \%$ (metaldehyde), while the 248 inter-day precision varied from $11.4 \%$ to $24.3 \%$ (pendimethalin). Intra and inter-day accuracy values were close to $100 \%$ (Table S1). 


\subsubsection{Detection and Quantification limits}

252 Limits of detection (Equation 1) and quantification (Equation 2) were calculated using the 253 standard deviation of the response and the slope, as described by ICH validation of analytical 254 procedures:

$$
L O D=3.3 \times \frac{\sigma_{R}}{m}
$$

$$
L O Q=10 \times \frac{\sigma_{R}}{m}
$$

257 where $\sigma_{R}$ is the standard deviation of the response and $m$ is the slope of the calibration curve.

258 The standard deviation of the response was calculated from the standard deviation of $y$ intercepts in the regression lines fitted to the data. Limits of detection and quantification ranged from 0.05 to $0.3 \mu \mathrm{g} \mathrm{L}^{-1}$ and 0.2 to $1.0 \mu \mathrm{g} \mathrm{L}^{-1}$, respectively (Table 3).

Table 3. Calibration curves, coefficient of determination $\left(\mathrm{r}^{2}\right)$, limit of detection $\left(\mu \mathrm{g} \mathrm{L} \mathrm{L}^{-1}\right)$ and limit of

\begin{tabular}{|c|c|c|c|c|c|}
\hline \multirow{2}{*}{ Analyte } & \multicolumn{2}{|c|}{ Calibration curve } & \multirow{2}{*}{$\mathbf{r}^{2}$} & \multirow{2}{*}{$\begin{array}{c}L O D \\
\left(\mu \mathrm{g} \mathrm{L}^{-1}\right)\end{array}$} & \multirow{2}{*}{$\begin{array}{c}L O Q \\
\left(\mu g \mathrm{~L}^{-1}\right)\end{array}$} \\
\hline & Slope & Intercept & & & \\
\hline Metaldehyde & $2219.7 \pm 15.3$ & 168.9 & 0.9998 & 0.09 & 0.3 \\
\hline Quinmerac & $2489.1 \pm 17.3$ & 45.9 & 0.9998 & 0.08 & 0.3 \\
\hline Carbetamide & $5524.8 \pm 33.9$ & 289.9 & 0.9998 & 0.09 & 0.3 \\
\hline Metazachlor & $11302 \pm 47.1$ & 584.1 & 0.9999 & 0.09 & 0.3 \\
\hline Propyzamide & $4544.5 \pm 72.9$ & 628.3 & 0.9987 & 0.05 & 0.2 \\
\hline Pendimethalin & $4636.1 \pm 154.8$ & 223.7 & 0.9944 & 0.3 & 1.0 \\
\hline
\end{tabular}
quantification $\left(\mu \mathrm{g} \mathrm{L}^{-1}\right)$. 


\subsubsection{Matrix effects}

268 To assess the matrix effect the slopes of the calibration curves for ultra-pure water (1) and stream water (2) were compared using a Student's $t$ test (95\%). The calculated value of $t, t_{c a l}$,

270 is defined by :

$271 \quad t_{c a l}=\frac{\left|b_{1}-b_{2}\right|}{\sqrt{S_{b 1}^{2}-S_{b 2}^{2}}}$

272

273 where $b$ is the slope of the calibration line and $S_{b}$ is the deviation of the slope.

274 The null hypothesis (there is no significant difference between the two calibration lines) was 275 rejected when $t_{c a l}$ was greater than the theoretical value $t_{\text {theo }} 2.306(p=0.05)$. Values of $t_{\text {cal }}$ 276 ranged from 0.5 to 1.3 for the different pesticides so that no significant matrix effect was 277 found. After approximately 80 samples, the mass spectrometer sensitivity was observed to 278 gradually decrease over time, probably because of deposition and accumulation of salts on 279 the cone surface. Analytical controls were used to identify when this problem occurred. 280 When sensitivity reduced by $15 \%$, the run was interrupted and maintenance was carried out.

\subsubsection{Blanks}

283 Ultra-pure water and methanol were used as solvent blanks during method validation and field sample analysis. No carryover or system peaks were found. Additionally, target analytes were undetected in field blanks.

\section{Applications of the method}

The method developed here has similar aims to those previously mentioned $[16,17]$ in that the main purpose is to detect multiple pesticides in environmental waters and to do this down to low levels around, $0.1 \mu \mathrm{g} / \mathrm{L}$. The main difference and indeed benefit of the method described in this paper is that an extraction is not needed and so large numbers of samples can be processed in a minimal timeframe, this also means that lower volumes of samples are needed resulting in less waste being developed and therefore a more efficient process. In addition to this, any potential errors that may occur during extraction processes are avoided. Data for stream discharge and stream water concentrations of the six pesticides analysed in water samples collected from the study stream are shown in Figure 3, between August and

298 December 2014. Daily rainfall data are also displayed. Pesticide concentrations tended to 
299 increase sharply during rainfall events with the highest concentrations typically occurring in 300 the first storm event after application. This is consistent with observations reported elsewhere 301 from catchments with under-drained heavy clay soils [3]. The highest concentrations were 302 observed for metaldehyde over an event in late August which triggered a relatively low 303 hydrograph peak. For quinmerac, which is applied later than metaldehyde, the first peak 304 concentrations occur in an event around the $13^{\text {th }}$ of October. Metaldehyde concentrations 305 also increase in this event but with lower peaks. Other notable increases in concentration 306 occur for carbetamide in a series of hydrographs starting on the $14^{\text {th }}$ of November and for propyzamide in the event of the $11^{\text {th }}$ of December, which also resulted in increases in

308 pendimethalin concentrations. Both propyzamide and carbetamide tend to be applied a little 309 later than some of the other herbicides due to the specific requirements of weed control 310 timing for blackgrass on oilseed rape. Concentrations of metazachlor were consistently low, 311 peaking at $0.37 \mu \mathrm{g} \mathrm{L}^{-1}$ on the $29^{\text {th }}$ of October. The magnitude of peak concentrations will 312 reflect a combination of factors including usage rate and the physico-chemical properties of 313 the compound. Compounds with high values of $K_{O C}$ (such as pendimethalin) will tend to 314 bind to soil solids and hence have a lower propensity to leach than compounds which are 315 more hydrophilic (such as metazachlor, quinmerac and carbetamide). For most compounds, 316 peak concentrations were observed at the same time as the hydrograph peak or slightly after 317 the peak flow (i.e. on the falling limb of the hydrograph), although apparent delays in the 318 appearance of peak pesticide concentration may be artefacts of the relatively low sampling frequency adopted $(8 \mathrm{~h})$.

321 Concentrations for all the pesticide compounds examined tended to decrease in hydrograph 322 recession periods in parallel with falling flow. Again, this is consistent with previous 323 observations of pesticide behaviour during storm events [3]. Clearly, peak concentrations of 324 all six pesticides were periodically greater than the maximum admissible concentration for 325 drinking water. Although this stream is not directly abstracted for water supply, it does feed into the River Great Ouse system, which is used for municipal abstraction downstream. The important point to note for the purposes of this paper is that the temporal pattern and magnitude of observed concentrations is consistent with expectations under the environmental conditions experienced over the study period. 


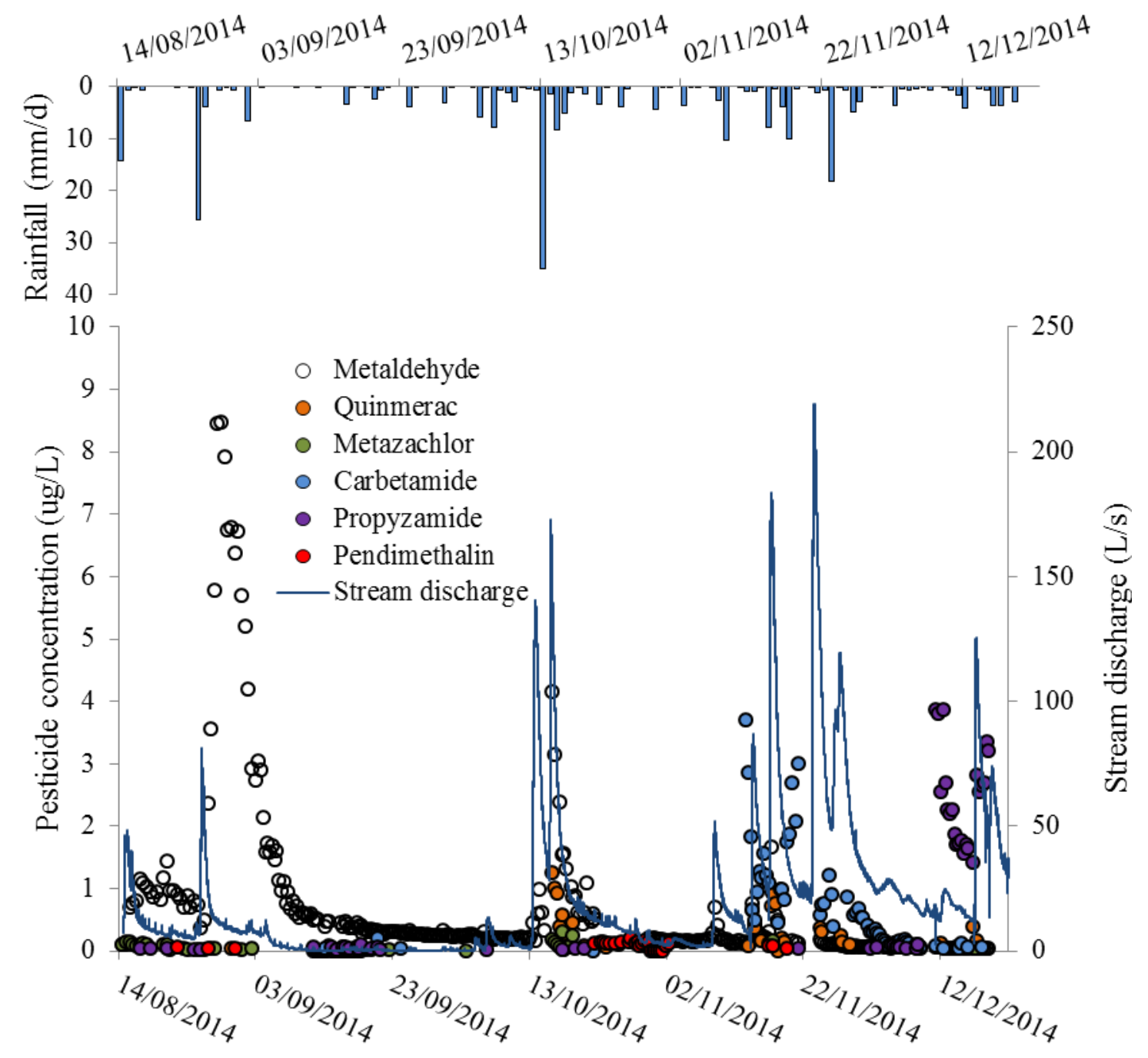

Figure 3. Rainfall (top panel), stream discharge (right axis) and pesticide concentrations (left axis) in the Hope Farm stream from August to December 2014. 


\section{Conclusions}

336 An LC-MS/MS method for the simultaneous multi-residue analysis of six pesticide active ingredients in natural waters is presented in this paper. This DAI method is rapid and accurate and can be used for quantification and confirmation of metaldehyde, quinmerac, carbetamide, metazachlor, propyzamide and pendimethalin in water samples from ground and surface waters. The omission of a concentration and clean-up step means that sample processing is fast and straightforward. The method showed a good range of linearity $\left(\mathrm{R}^{2}\right.$ ranged from 0.995 to 0.999 ), accuracy ( 84 to $100 \%$ ) and RSD precision (4 to $15 \%$ ) and there was negligible apparent matrix effect compared to the same pesticides in ultra-pure water.

345 The LOQs obtained ranged from 0.2 to $1.0 \mu \mathrm{g} \mathrm{L}^{-1}$. This is acceptable for detecting concentrations in natural water samples from many agricultural catchments where pesticide concentrations are high (edge of field concentrations often exceed $100 \mu \mathrm{g} \mathrm{L}^{-1}$ [3]) but would

348 be of limited value in assessing DWD compliance. The use of a multi-residue method with rapid and simple sample preparation reduces analysis time and improves laboratory efficiency. The temporal pattern and magnitude of concentrations in samples from a headwater arable stream were consistent with expectations for the environmental conditions experienced over the study period, suggesting that the method can yield a realistic description of pesticide exposure in natural waters.

\section{Acknowledgements}

357 This work was funded jointly by the Chemicals Regulation Directorate of the UK Health and 358 Safety Executive and Lonza with additional support from Severn Trent Water Limited. We 359 would like to thank the RSPB for granting access to Hope Farm for sample collection, Ian

360 Dillon (RSPB) for providing information on pesticide use and Ian Baylis and Vassia 361 Ioannidou (University of Warwick) for help with weir installation and monitoring. MW benefitted from Study Leave granted by the University of Leicester. 
365

366

367

368

369

370

371

372

373

374

375

376

377

378

379

380

381

382

383

384

385

386

387

388

389

390

391

\section{References}

[1] T. Dolan, P. Howsam, D. J. Parsons, and M. J. Whelan, "Is the EU drinking water directive standard for pesticides in drinking water consistent with the precautionary principle?," Environ. Sci. Technol., vol. 47, no. 10, pp. 4999-5006, 2013.

[2] J. Tournebize, E. Passeport, C. Chaumont, C. Fesneau, a. Guenne, and B. Vincent, "Pesticide de-contamination of surface waters as a wetland ecosystem service in agricultural landscapes,” Ecol. Eng., vol. 56, pp. 51-59, Jul. 2013.

[3] A. Tediosi, M. J. Whelan, K. R. Rushton, T. R. E. Thompson, C. Gandolfi, and S. P. Pullan, "Measurement and conceptual modelling of herbicide transport to field drains in a heavy clay soil with implications for catchment-scale water quality management.," Sci. Total Environ., vol. 438, pp. 103-12, Nov. 2012.

[4] C. Li, Y.-L. Wu, T. Yang, and Y. Zhang, "Determination of Metaldehyde in Water by SPE and UPLC-MS-MS,” Chromatographia, vol. 72, no. 9-10, pp. 987-991, 2010.

[5] M. J. Whelan, R. Van Egmond, I. Guymer, J. O. Lacoursi??re, L. M. B. Vought, C. Finnegan, K. K. Fox, C. Sparham, S. O’Connor, M. Vaughan, and J. M. Pearson, “The behaviour of linear alkyl benzene sulphonate under direct discharge conditions in Vientiane, Lao PDR,” Water Res., vol. 41, no. 20, pp. 4730-4740, 2007.

[6] M. T. Wan, J. Kuo, and J. Pasternak, "Residues of endosulfan and other selected organochlorine pesticides in farm areas of the Lower Fraser Valley, British Columbia, Canada.," J. Environ. Qual., vol. 34, no. 4, pp. 1186-1193, 2005.

[7] C. Hao, D. Morse, X. Zhao, and L. Sui, "Liquid chromatography/tandem mass spectrometry analysis of neonicotinoids in environmental water," Rapid Commun. Mass Spectrom., vol. 29, no. 23, pp. 2225-2232, 2015.

[8] P. Kay and R. Grayson, "Using water industry data to assess the metaldehyde pollution problem," vol. 28, pp. 410-417, 2014.

[9] B. Tao and A. J. Fletcher, "Metaldehyde removal from aqueous solution by adsorption and ion exchange mechanisms onto activated carbon and polymeric sorbents," $J$. 
393

[10] R. Romero-González, A. G. Frenich, and J. L. M. Vidal, "Multiresidue method for fast determination of pesticides in fruit juices by ultra performance liquid chromatography coupled to tandem mass spectrometry," Talanta, vol. 76, no. 1, pp. 211-225, 2008.

[11] A. Stachniuk and E. Fornal, "Analytical considerations on the use of a fruit-specific and representative matrix in pesticide residue analysis by LC-ESI-MS/MS," Cent. Eur. J. Chem., vol. 11, no. 7, pp. 1112-1131, 2013.

[12] Y. Ono, T. Yamagami, T. Nishina, and T. Tobino, "Pesticide multiresidue analysis of 303 compounds using supercritical fluid extraction.," Anal. Sci., vol. 22, no. 11, pp. 1473-6, 2006.

[13] J. Fenoll, P. Hellín, C. M. Martínez, M. Miguel, and P. Flores, "Multiresidue method for analysis of pesticides in pepper and tomato by gas chromatography with nitrogenphosphorus detection," Food Chem., vol. 105, no. 2, pp. 711-719, 2007.

[14] C. Crescenzi, A. Di Corcia, E. Guerriero, and R. Samperi, "Development of a multiresidue method for analyzing pesticide traces in water based on solid-phase extraction and electrospray liquid chromatography mass spectrometry," Environ. Sci. Technol., vol. 31, no. 2, pp. 479-488, 1997.

[15] J. Quintana, I. Martí, and F. Ventura, "Monitoring of pesticides in drinking and related waters in NE Spain with a multiresidue SPE-GC-MS method including an estimation of the uncertainty of the analytical results," J. Chromatogr. A, vol. 938, no. 1-2, pp. 313, 2001.

[16] S. Irace-Guigand, J. J. Aaron, P. Scribe, and D. Barcelo, "A comparison of the environmental impact of pesticide multiresidues and their occurrence in river waters surveyed by liquid chromatography coupled in tandem with UV diode array detection and mass spectrometry," Chemosphere, vol. 55, no. 7, pp. 973-981, 2004.

[17] H. G. J. Mol, A. Rooseboom, R. Van Dam, M. Roding, K. Arondeus, and S. Sunarto, "Modification and re-validation of the ethyl acetate-based multi-residue method for pesticides in produce," Anal. Bioanal. Chem., vol. 389, no. 6, pp. 1715-1754, 2007.

[18] Ea, "Environment Agency The determination of metaldehyde in waters using 
chromatography with mass spectrometric detection (2009)," 2009.

422 [19] ICH Harmonised Tripartite Guideline - "Validation of Analytical Procedures: Text and 423 Methodology", 2005, http://www.ich.org 